\title{
Neferine inhibits angiotensin II-stimulated proliferation in vascular smooth muscle cells through heme oxygenase-1
}

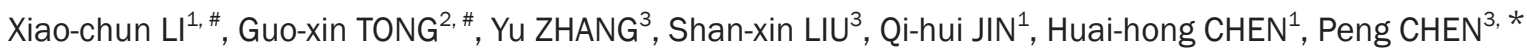 \\ ${ }^{1}$ Department of Geratology, the Second Affiliated Hospital, School of Medicine, Zhejiang University, Hangzhou 310009, China; \\ ${ }^{2}$ Department of Cardiology, the First People's Hospital of Hangzhou, Hangzhou 310006, China; ${ }^{3}$ Department of Cardiology, the First \\ Affiliated Hospital, School of Medicine, Zhejiang University, Hangzhou 310003, China
}

\begin{abstract}
Aim: To explore the effect of neferine on angiotensin II (Ang II)-induced vascular smooth muscle cell (VSMC) proliferation.
Methods: Human umbilical vein smooth muscle cells (HUVSMCs) were used. Cell proliferation was determined by using the 3-(4,5-dimethylthiazol-2-yl)-2,5-diphenyltetrazolium bromide (MTT) assay and flow cytometry analysis. Heme oxygenase (HO)-1 protein expression was tested by Western blot analysis. Extracellular signal-regulated protein kinase 1/2 (ERK1/2) activation was determined by using immunoblotting.

Results: Pre-incubation of HUVSMCs with neferine (0.1, 0.5, 1.0, and $5.0 \mu \mathrm{mol} / \mathrm{L})$ significantly inhibited Ang II-induced cell proliferation in a concentration-dependent manner and neferine $5.0 \mu \mathrm{mol} / \mathrm{L}$ increased HO-1 expression by $259 \%$ compared with control. The antiproliferative effect of neferine was significantly attenuated by coapplication of zinc protoporphyrin IX (ZnPP IX, an HO-1 inhibitor) with neferine. Ang II-enhanced ERK1/2 phosphorylation was markedly reversed by neferine. By inhibiting HO-1 activity with ZnPP IX, the inhibitive effect of neferine on ERK1/2 phosphorylation was significantly attenuated. Cobalt-protoporphyrin (CoPP), an HO-1 inducer, significantly decreased Ang II-induced ERK1/2 phosphorylation and inhibited Ang II-induced cell proliferation. The ERK1/2 pathway inhibitor PD98059 significantly blocked Ang II-enhanced ERK1/2 phosphorylation and inhibited cell proliferation.

Conclusion: These findings suggest that neferine can inhibit Ang II-induced HUVSMC proliferation by upregulating HO-1, leading to the at least partial downregulation of ERK1/2 phosphorylation.
\end{abstract}

Keywords: neferine; heme oxygenase-1; angiotensin II; zinc protoporphyrin; vascular smooth muscle cells; cell proliferation

Acta Pharmacologica Sinica (2010) 31: 679-686; doi: 10.1038/aps.2010.57

\section{Introduction}

The proliferation and migration of vascular smooth muscle cells (VSMCs) play a pivotal role in the pathogenesis of atherosclerosis and restenosis after angioplasty and possibly in the development of hypertension. A vital stimulus for VSMC proliferation is angiotensin II (Ang II), the main effector of the renin-angiotensin system. $\mathrm{AT}_{1}$, an Ang II receptor, mediates most of the physiological and pathophysiological actions of Ang II. Activation of $\mathrm{AT}_{1}$ stimulates various signaling molecules, including protein kinase $\mathrm{C}$, mitogen-activated protein kinase (MAPK), and tyrosine kinases ${ }^{[1,2]}$. Moreover, activation of tyrosine kinases by Ang II also leads to the activation

\footnotetext{
\# These authors contributed equally to this work.

* To whom correspondence should be addressed.

E-mail pchen_helen@163.com

Received 2010-01-24 Accepted 2010-04-08
}

of MAPK and consequently promotes the growth response in VSMCs ${ }^{[3,4]}$. Ang II is known to stimulate the activation of MAPKs, including extracellular signal-regulated protein kinase (ERK) 1/2, p38MAPK, and stress-activated protein kinase/c-Jun N-terminal kinase (JNK) in VSMCs. The ERK pathway is the best characterized in the MAPK pathways. Binding of Ang II to $\mathrm{AT}_{1}$ activates ERK1/2 within $5 \mathrm{~min}^{[2]}$. Among the MAP kinase family, ERK1/2 has been implicated in the proliferation of various cell types ${ }^{[5]}$. Furthermore, Ang II-induced activation of ERK1/2 has also been implicated in hypertension and in micro- and macrovascular target-organ damage.

Heme oxygenase $(\mathrm{HO})$ catalyzes heme degradation into carbon monoxide (CO), iron, and biliverdin. $\mathrm{HO}$ is a microsomal enzyme with an inducible isoform $\mathrm{HO}-1$ and constitutive forms HO-2 and HO-3. All HO isoforms are inhibited by the metallo-protoporphyrins zinc protoporphyrin IX (ZnPP 
IX) and tin protoporphyrin IX (SnPP IX). $\mathrm{HO}$ is expressed in many types of cells, including VSMCs ${ }^{[6]}$. HO-1, also known as heat shock protein 32 , is ubiquitously distributed and highly inducible by a variety of physiological and pathophysiological stimuli $^{[7]}$. Accumulated evidence indicates that HO-1 offers protection against many vascular disorders. Increased HO-1 expression reduces intimal thickening after arterial injury and attenuates atherosclerosis in apolipoprotein E deficient mice ${ }^{[8]}$. Moreover, increased activity of HO-1 inhibits proliferation of $\mathrm{VSMCs}^{[9-11]}$. Interestingly, it has recently been reported that HO-1 inhibits the proliferation of pancreatic stellate cells by repression of the ERK1/2 pathway ${ }^{[12]}$.

Many traditional Chinese medicines used for treating cardiovascular disorders are very effective. Among the complex chemical ingredients of traditional Chinese medicines, there usually is a major ingredient that can be useful for the treatment of cardiovascular disorders; these ingredients provide an obvious starting point for the development of new drugs for the treatment of cardiovascular disorders, as well as for defining new pathways involved in the pathogenesis of cardiovascular diseases. Neferine (Nef), a major biologically active compound, is extracted from the green seed embryo of Nelumbo nucifera Gaertn. Neferine is a bis-benzylisoquinoline alkaloid, and its chemical structure is shown in Figure 1. A wide range of biological and pharmacological actions of neferine have been investigated ${ }^{[13,14]}$. It has been proposed that neferine can prevent foam cell formation by inhibiting the oxygenation of very low density lipoprotein and platelet aggregation ${ }^{[15,16]}$. Moreover, neferine can protect vascular endothelial cells from damage induced by oxygen free radicals. In addition, neferine is effective in preventing the onset of reentrant ventricular tachyarrhythmias ${ }^{[17]}$. These findings indicate that neferine has protective effects on the cardiovascular system. However, the effect of neferine on VSMC proliferation, the key event in the pathogenesis of atherosclerosis and restenosis, has remained unclear.

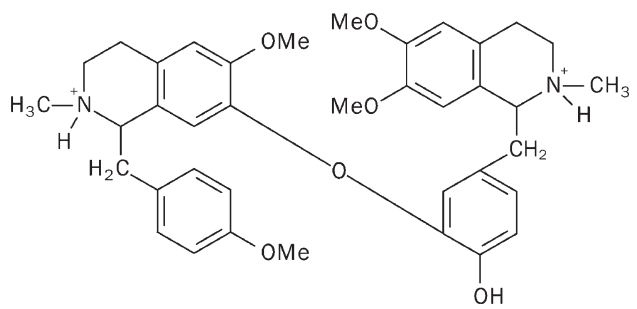

Figure 1. Molecular structure of neferine.

The aim of this study was to examine whether neferine would affect Ang-induced VSMC proliferation through HO-1 expression and to investigate the underlying signaling pathways.

\section{Materials and methods Materials}

Dulbecco's modified Eagle's medium (DMEM) with high glu- cose and fetal bovine serum (FBS) were purchased from Gibco BRL (Grand Island, NY, USA) and Hyclone, respectively. Proprep protein extract solution was purchased from Beyotime Institute of Biotechnology (Shanghai, China). The polyclonal anti-phospho-extracellular signal-regulated kinase (ERK)1/2 and anti-total-ERK1/2 rabbit antibodies were obtained from Cell Signaling Technology Inc. Polyclonal rabbit anti-HO-1 and monoclonal mouse anti-glyceraldehyde 3-phosphate dehydrogenase (GAPDH) antibodies were obtained from R\&D Systems, Inc (Minneapolis, MN, USA). The mitogen/extracellular signal-regulated kinase (MEK) inhibitor PD98059 was supplied by Calbiochem (Bad Soden, Germany). 3-(4,5-Dimethylthiazol-2-yl)-2,5-diphenyltetrazolium (MTT) was purchased from Sigma-Aldrich (St Louis, MO, USA). Ang II and Zinc Protoporphyin IX (Znpp IX) were purchased from Merck \& Co, Inc (Whitehouse Station, NJ, USA). Cobalt-protoporphyrin (CoPP) was from Porphyrin Products (London, UK). Neferine was purchased from Phytomarker Ltd (Tianjin, China). The purity of Nef was greater than $99.8 \%$.

Stock solutions of ZnPP IX and neferine were prepared in a small volume of $100 \%$ dimethyl sulfoxide (DMSO, equivalent to $<1 \%$ of the final volume). Ang II was dissolved in methanol. The solution was filtered through a $0.22-\mu \mathrm{m}$ membrane. Aliquots were stored at $-20{ }^{\circ} \mathrm{C}$ in the dark. Further dilution was made with cell culture medium. All other reagents were purchased from Sigma-Aldrich.

\section{Cell culture}

The primary cell line of Human Umbilical Vein Smooth Muscle Cells (HUVSMC) was obtained from the Health Protection Agency (HPA) Culture Collections (UK). HUVSMCs were grown in DMEM supplemented with $10 \%$ heat-inactivated FBS. The cells were maintained at $37{ }^{\circ} \mathrm{C}$ in a $95 \% \mathrm{O}_{2} / 5 \% \mathrm{CO}_{2}$ incubator. The medium was changed every $2 \mathrm{~d}$. Cells were digested with $0.25 \%$ trypsin and $0.02 \%$ EDTA when the cell density reached $80 \%$ to $90 \%$. HUVSMCs from 4 to 8 passages at $70 \%-90 \%$ confluence were used in 6-well cell culture dishes. HUVSMCs were incubated in serum-free medium for $24 \mathrm{~h}$ before the addition of experimental reagents.

\section{Western blot analysis}

HUVSMCs were plated on 6-well culture plates in 10\% FBS/ DMEM and grown to $80 \%$ confluence. After $24 \mathrm{~h}$ of quiescence in serum-free medium, cells were treated as indicated. Total cell lysates were prepared by the addition of $100 \mu \mathrm{L}$ of proprep protein extract solution. The protein concentration was quantified with a protein assay reagent from Bio-Rad (Hercules, CA, USA). Equal amounts of protein were mixed with sodium dodecyl sulfate (SDS) sample buffer and incubated for $5 \mathrm{~min}$ at $100{ }^{\circ} \mathrm{C}$ before loading. Total protein samples $(40 \mu \mathrm{g})$ were subjected to $10 \%$ SDS-polyacrylamide gel electrophoresis $\left(\right.$ SDS-PAGE) ${ }^{[18]}$ for $2 \mathrm{~h}$ at $100 \mathrm{~V}$. The separated proteins were electrophoretically transferred onto a PVDF membrane for $1 \mathrm{~h}$ at $30 \mathrm{~mA}$. Membranes were blocked with 5\% non-fat milk in phosphate buffered saline containing 0.05\% Tween 20 (PBS-T) for $2 \mathrm{~h}$ at room temperature. Membranes were incubated with 
the primary antibodies at a dilution of 1:1000 in 1\% bovine serum albumin (BSA) overnight at $4{ }^{\circ} \mathrm{C}$. The membranes were washed four times with PBS-T and then probed with IRDye ${ }^{\circledR}$ $800 \mathrm{CW}$ conjugated secondary antibodies. The IRDye $800 \mathrm{CW}$ activity was detected using a LI-COR Odyssey (LI-COR Biosciences, USA). For normalization, the total amount of GAPDH was detected. All results shown are representative of experiments with at least three different cell preparations.

\section{Cell proliferation assay}

Cell proliferation was analyzed using the MTT assay ${ }^{[19]}$. HUVSMCs were seeded on 96-well plates at a density of $0.6-1.0 \times 10^{4}$ cells per well in DMEM supplemented with $10 \%$ FBS. Serum-free DMEM was added to each well after $24 \mathrm{~h}$ and incubated for $24 \mathrm{~h}$ to synchronize cells to the resting stage. The supernatant was removed, and DMEM and 1\% FBS containing Ang II, Nef, and ZnPP IX were added and co-cultured for $24 \mathrm{~h}$. This was repeated five times for each concentration group. After treatments with Ang II, neferine and HO-1 inhibitor, $20 \mu \mathrm{L}$ of $5 \mathrm{mg} / \mathrm{mL}$ MTT solution was added to each well and incubated for $4 \mathrm{~h}$. The supernatants were aspirated, and the formazan crystals in each well were dissolved with 150 $\mu \mathrm{L}$ DMSO. Cell proliferation was assessed by measuring the absorbance at $490 \mathrm{~nm}$ using a microplate reader. The experiments were repeated three times.

\section{Cell cycle analysis}

Cell cycle status was analyzed with a FACScan flow cytometer (Becton Dickinson, San Diego, USA) by measuring fluorescence from cells stained with propidium iodide ${ }^{[20]}$. Data are presented as the relative fluorescence intensity of cell sub-populations in the 2-dimensional FACS profile or as the percentage of cells in a given sub-population. Cells were stained with $10 \mu \mathrm{g} / \mathrm{mL}$ propidium iodide (PI) solution and RNaseA (Santa Cruz Biotechnology) in PBS in the dark for at least $30 \mathrm{~min}$ at room temperature. At least 10000 cells were analyzed in each sample. The percentages of cells in the different phases of the cell cycle were measured with a FACS flow cytometer and analyzed with ModFit 3.0 software.

\section{Statistical analysis}

Results are expressed as means \pm SEM for the indicated number of separate cell preparations per experimental protocol. Data were analyzed using one-way analysis of variance (ANOVA) followed by the Student Newman-Keuls post hoc Tukey test. Differences between groups were considered significant at $P<0.05$. Analyses were performed with the SPSS statistical software package (15.0).

\section{Results}

\section{Neferine inhibited Ang II-induced proliferation of HUVSMCs}

First, HUVSMCs were cultured in medium supplemented with Ang II $(0.001,0.01,0.1$, and $1.0 \mu \mathrm{mol} / \mathrm{L})$ for $24 \mathrm{~h}$. As shown in Figure 2A, exposure of HUVSMC to Ang II resulted in a significant increase in the number of cells, with maximal levels at 0.1 $\mu \mathrm{mol} / \mathrm{L}$. Ang II at $0.1 \mu \mathrm{mol} / \mathrm{L}$ significantly increased prolif- eration by $51 \%$ compared with controls. Cytotoxicity was not observed at $0.1 \mu \mathrm{mol} / \mathrm{L}$. Therefore, the $0.1 \mu \mathrm{mol} / \mathrm{L}$ concentration was selected for further studies. Compared to the control group, neferine $(0.1,0.5,1.0$, and $5.0 \mu \mathrm{mol} / \mathrm{L})$ decreased cell proliferation in a concentration-dependent manner, reaching a maximal response at $5.0 \mu \mathrm{mol} / \mathrm{L}$. HUVSMC proliferation was significantly inhibited by neferine $(5 \mu \mathrm{mol} / \mathrm{L})$ at 24 and $48 \mathrm{~h}$, being $18 \%$ and 19\% lower, respectively, than controls (Figure 2B). The concentrations used for neferine did not exhibit cytotoxic effects on cell viability; therefore, the $5.0 \mu \mathrm{mol} / \mathrm{L}$ concentration of neferine was used in the following studies. As shown in Figure 2C, proliferation of HUVSMC incubated with Ang II was significantly attenuated in the presence of neferine, suggesting that neferine has a potential antiproliferative effect on HUVSMC stimulated by Ang II. The antiproliferative effect of neferine was confirmed by cell cycle analysis. As described in Figure 2D, the rate of cells entering into mitosis can be estimated from the proliferation index. After $24 \mathrm{~h}$ of stimulation with Ang II, the percentage of $\mathrm{G}_{2} / \mathrm{M}$ cells increased from $14.42 \%$ to $25.33 \%$. During the same time period in the presence of neferine $(5.0 \mu \mathrm{mol} / \mathrm{L})$, the rate of entry into mitosis of cells exposed to Ang II was reduced from $25.33 \%$ to $13.29 \%$. The cell proliferation index refers to the percentage of cells in $S$ phase and $G_{2} / M$ phase, and is a major indicator of proliferation of the cell population. After stimulation with Ang II, the proliferation index increased by $93 \%$ compared with controls, but in the presence of neferine $(5 \mu \mathrm{mol} / \mathrm{L})$, the proliferation index of cells exposed to Ang II was decreased to $85 \%$ of controls. ZnPP IX significantly abolished the inhibitory effect of neferine on the proliferation index. The change in the percentage of cells in $S$ phase was in accordance with the above observation. Neferine arrested HUVSMCs at the $\mathrm{G}_{0} / \mathrm{G}_{1}$ phase.

\section{Neferine increased HO-1 protein expression in HUVSMC stimulated by Ang II}

The results shown in Figure 3A indicated that $5 \mu \mathrm{mol} / \mathrm{L}$ neferine significantly increased $\mathrm{HO}-1$ expression by $259 \%$ compared with controls. The presence of Ang II alone significantly inhibited HO-1 expression by $44 \%$ compared with controls, whereas neferine increased Ang II-inhibited HO-1 expression by $125 \%$ in HUVSMCs (Figure 3B). Neferine not only remarkably induced the expression of HO-1 in a concentration-dependant manner when compared with controls, but also significantly increased the expression of HO-1 in cells inhibited by Ang II (Figure 3).

\section{Neferine inhibited Ang II-induced HUVSMC proliferation through HO-1}

Considering that neferine caused the up-regulation of HO-1 expression, whether neferine-induced $\mathrm{HO}-1$ expression could facilitate its antiproliferative effect was tested. It was found that coapplication of neferine and ZnPP IX $(10 \mu \mathrm{mol} / \mathrm{L})$, a HO-1 activity inhibitor, had no significant effect on neferine upregulation of HO-1 expression in HUVSMCs treated with Ang II (Figure 3B); however, as shown in Figure 2C, the inhibitory effect of neferine on Ang II-induced cell proliferation 
A

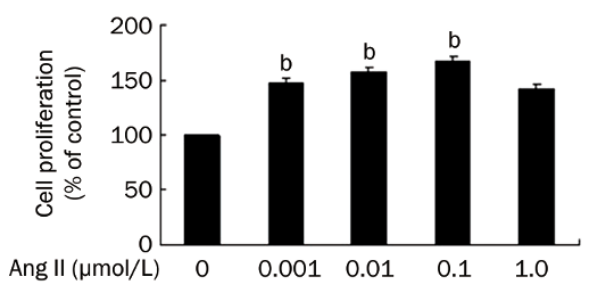

B

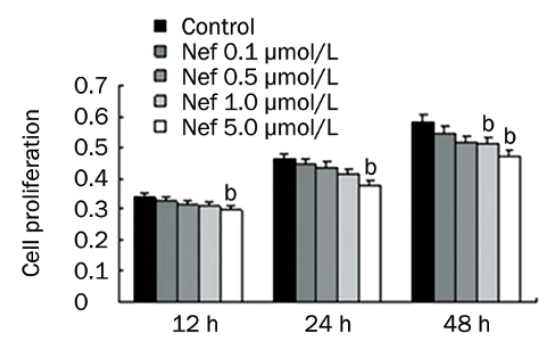

C

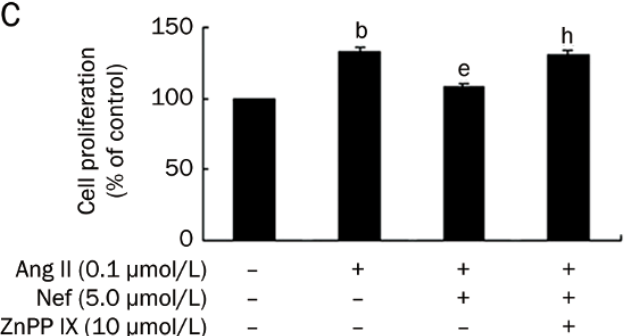

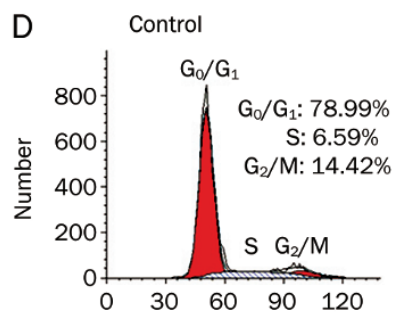
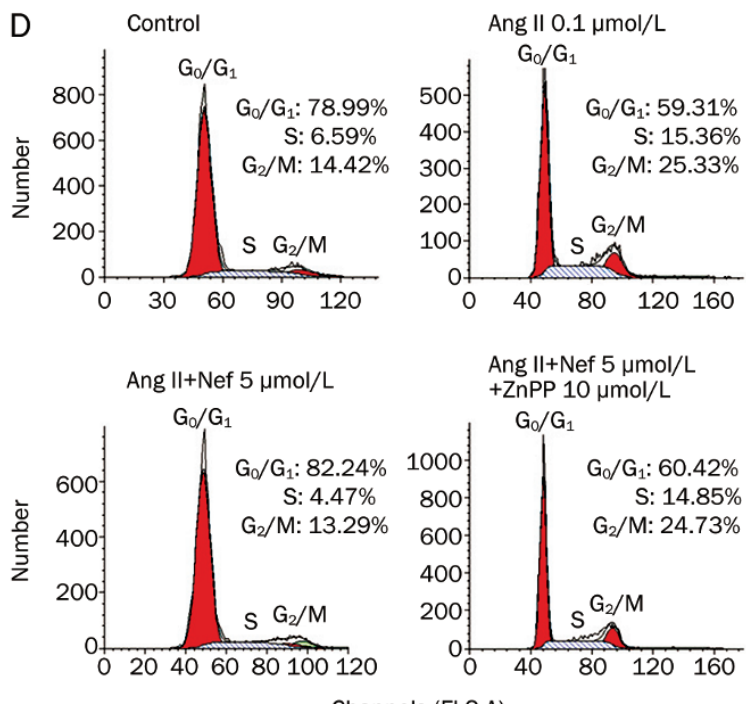

Channels (FL2-A)

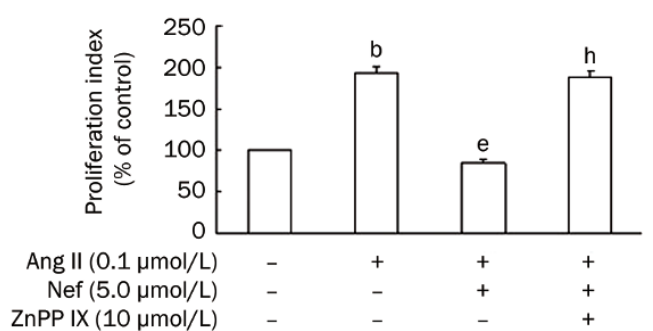

Figure 2. Neferine inhibited Ang Il-induced proliferation of HUVSMC. (A) HUVSMC proliferation was determined using the MTT assay. Cells were treated with Ang II (0, 0.001, 0.01, 0.1, and $1.0 \mu \mathrm{mol} / \mathrm{L})$ for $24 \mathrm{~h}$. (B) The effect of neferine on HUVSMC proliferation in the presence of $1 \%$ FBS was investigated. Neferine $(0.1-5.0 \mu \mathrm{mol} / \mathrm{L})$ inhibited HUVSMC proliferation in a dose-dependent manner after 24 and $48 \mathrm{~h}$ compared with cells treated with cultured medium alone. (C) Neferine inhibited Ang II-stimulated cell proliferation by $25 \%$; the effect of neferine was reduced by pretreatment of cells with the HO inhibitor ZnPP IX (10 $\mu \mathrm{mol} / \mathrm{L})$. (D) Effect of Ang II and ZnPP IX in the presence or absence of neferine on the cell cycle in HUVSMCs. Cell cycle distribution was assessed by flow cytometry. HUVSMCs were treated with ZnPP IX for $1 \mathrm{~h}$ in the presence of neferine and then stimulated with Ang II for $24 \mathrm{~h}$. The cell proliferation index in the histogram refers to the percentage of cells in $S$ and $G_{2} / M$ phases. ${ }^{b} P<0.05$ compared with control; ${ }^{\mathrm{e}} \mathrm{P}<0.05$ compared with Ang II; ${ }^{\mathrm{h}} \mathrm{P}<0.05$ compared with Ang II+neferine.

was significantly reversed by ZnPP IX. Furthermore, CoPP, a HO-1 inducer, was capable of preventing Ang II-induced proliferation (Figure 4). Pre-incubation with CoPP (20 $\mu \mathrm{mol} / \mathrm{L}$ ) decreased Ang II-induced cell proliferation by $45 \%$ in HUVSMCs, whereas ZnPP IX ( $10 \mu \mathrm{mol} / \mathrm{L})$ increased it by $47 \%$. Pre-incubation with PD98059 (20 $\mu \mathrm{mol} / \mathrm{L})$ also decreased Ang II-induced cell proliferation by $50 \%$ in HUVSMCs. Moreover, PD98059 inhibited the increase in cell proliferation induced by ZnPP IX. These findings suggest that neferine-induced HO-1 expression and the resultant increase in activity of HO- 1 is involved in the antiproliferative effect of neferine on HUVSMC induced by Ang II.

Neferine-induced HO-1 expression modulated ERK1/2 phosphorylation

Ang II induced ERK1/2 phosphorylation in a time-dependent manner, reaching $296 \%$ of controls at the $120 \mathrm{~min}$ time-point (Figure 5A). The Ang II-enhanced ERK1/2 phosphorylation was markedly reversed by neferine (Figure 5B), which increased HO-1 expression in Ang II-stimulated cells (Figure $3 \mathrm{~B})$. Neferine significantly reduced phosphorylation of ERK $1 / 2$ induced by Ang II by $148 \%$. The application of ZnPP IX significantly attenuated the inhibitory effect of neferine on ERK 1/2 phosphorylation (Figure 5B). Moreover, CoPP, a HO-1 inducer, significantly decreased Ang II-induced ERK1/2 phosphorylation, suggesting that up-regulation of HO-1 represses ERK1/2 phosphorylation caused by Ang II. Preincubation with CoPP $(20 \mu \mathrm{mol} / \mathrm{L})$ decreased Ang II-induced ERK1/2 phosphorylation by $74 \%$ in HUVSMCs, whereas ZnPP IX $(10 \mu \mathrm{mol} / \mathrm{L})$ increased it by $77 \%$. Pre-incubation with PD98059 $(20 \mu \mathrm{mol} / \mathrm{L})$ also decreased Ang II-induced ERK1/2 phosphorylation by $52 \%$ in HUVSMCs. Moreover, PD98059 significantly inhibited ZnPP IX-induced ERK1/2 phosphorylation in HUVSMCs (Figure 5C).

\section{ERK1/2 phosphorylation modulated Ang II-induced HUVSMC} proliferation

Ang II increased ERK1/2 phosphorylation and proliferation in 
A
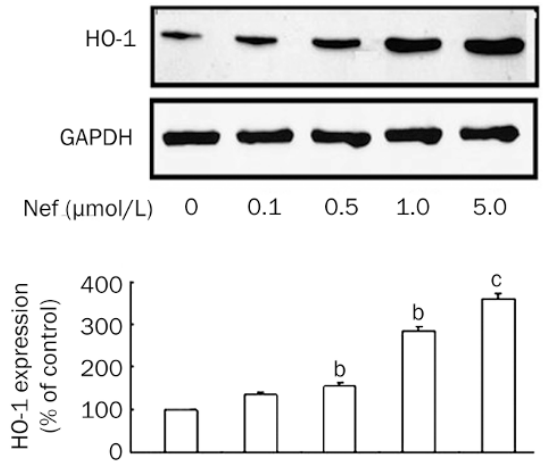

B
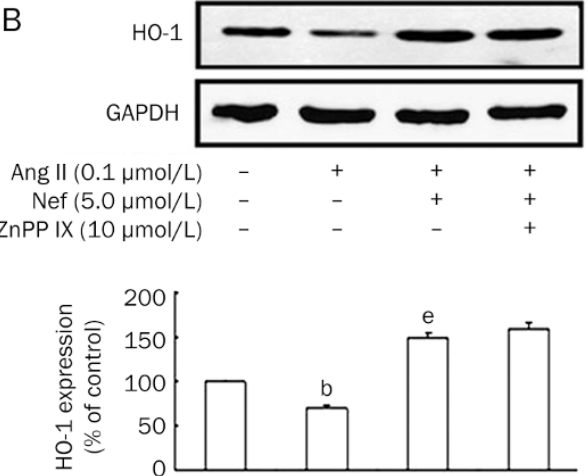

Figure 3. Nefrine increased HO-1 protein expression in HUVSMCs stimulated by Ang II. HUVSMCs treated with 1\% FBS served as controls. The results represent Western blot analysis for HO-1 protein expression. To verify equal loading, membranes were stripped and probed with an anti-GAPDH protein-specific antibody. (A) Cells were treated with neferine $(0,0.1,0.5,1.0$, and $5.0 \mu \mathrm{mol} / \mathrm{L})$ for $24 \mathrm{~h}$. Neferine concentrationdependently increased HO-1 expression. (B) Serum-starved HUVSMCs were treated with neferine $(5 \mu \mathrm{mol} / \mathrm{L})$ and $\mathrm{ZnPP} I X(10 \mu \mathrm{mol} / \mathrm{L})$ for $1 \mathrm{~h}$ then treated by a stimulation with Ang II $(0.1 \mu \mathrm{mol} / \mathrm{L})$ for $24 \mathrm{~h} .{ }^{\mathrm{b}} P<0.05$, ${ }^{\mathrm{c}} P<0.01$ compared with control. ${ }^{\mathrm{e}} P<0.05$ compared with Ang II.

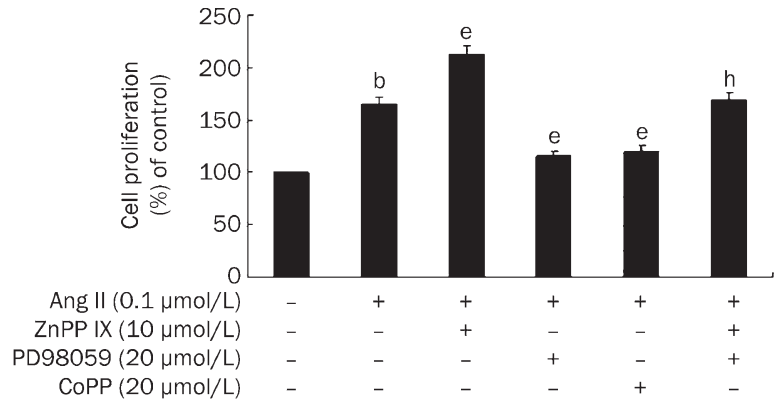

Figure 4. Neferine inhibited Ang II-induced HUVSMC proliferation through HO-1. Assessment of the roles of PD98059 (a ERK1/2 pathway inhibitor), ZnPP IX (a HO-1 inhibitor) and CoPP (a HO-1 inducer) on cell proliferation by MTT assay before a $24 \mathrm{~h}$ stimulation with Ang II. ${ }^{\mathrm{b}} P<0.05$ vs control. ${ }^{\mathrm{e}} P<0.05$ vs Ang II. ${ }^{\mathrm{h}} P<0.05$ vs Ang II+ZnPP IX.

HUVSMC (Figure 2C, 4, 5A-5C), whereas neferine decreased ERK1/2 phosphorylation and proliferation in HUVSMC
A

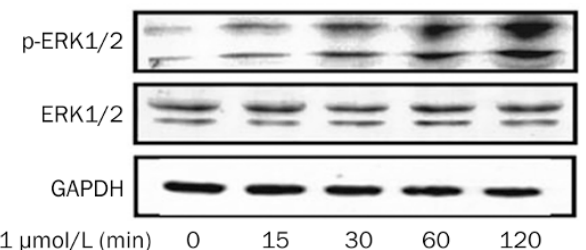

Ang II $0.1 \mu \mathrm{mol} / \mathrm{L}$ (min) $\quad 0 \quad 15 \quad 30 \quad 60 \quad 120$

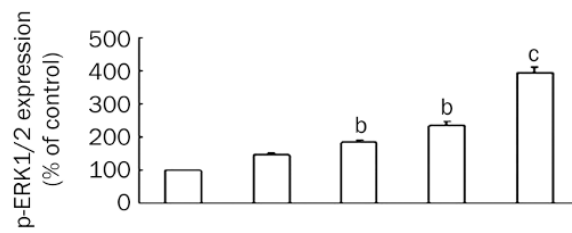

B

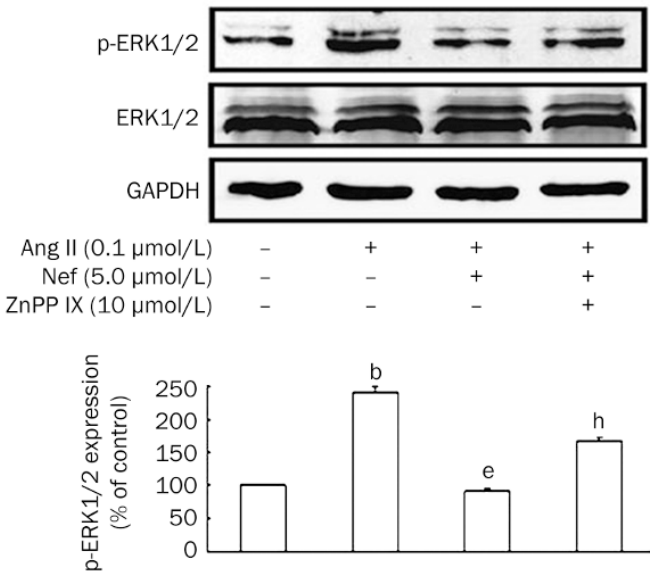

C
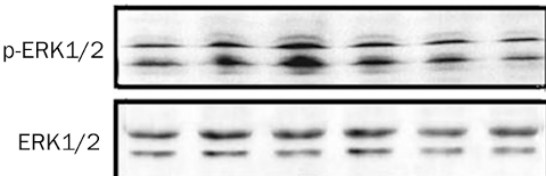

GAPDH

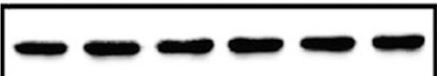

Ang II $(0.1 \mu \mathrm{mol} / \mathrm{L})$

ZnPP IX (10 $\mu \mathrm{mol} / \mathrm{L})$ PD98059 $(20 \mu \mathrm{mol} / \mathrm{L})$ CoPP $(20 \mu \mathrm{mol} / \mathrm{L})$

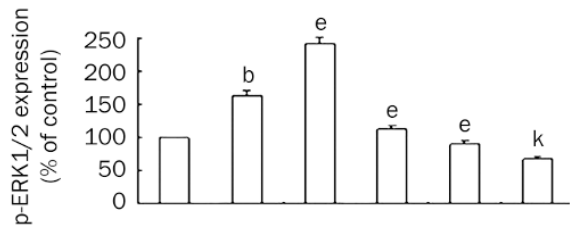

Figure 5. Neferine-induced HO-1 expression modulated ERK1/2 phosphorylation. Phosphorylated ERK1/2 (p-ERK1/2) levels were determined by Western blot analysis. (A) HUVSMCs were starved from serum for $24 \mathrm{~h}$ before stimulation with Ang II (0.1 $\mu \mathrm{mol} / \mathrm{L})$ for 15, 30, 60 , and $120 \mathrm{~min}$. (B) Serum-starved HUVSMCs were pretreated with neferine $(5 \mu \mathrm{mol} / \mathrm{L})$ and/or ZnPP IX $(10 \mu \mathrm{mol} / \mathrm{L})$ for $1 \mathrm{~h}$ before stimulation with Ang II $(0.1 \mu \mathrm{mol} / \mathrm{L})$ for $24 \mathrm{~h}$. (C) Western blot analysis of ERK1/2 phosphorylation after a 120 min stimulation with $0.1 \mu \mathrm{mol} / \mathrm{L}$ Ang II. The HUVSMCs were pretreated with different reagents for $1 \mathrm{~h}$ before stimulation. ${ }^{\mathrm{b}} P<0.05,{ }^{\mathrm{c}} P<0.01$ vs control. ${ }^{\mathrm{e}} P<0.05$ vs Ang II. ${ }^{\mathrm{h}} P<0.05$ vs Ang II+neferine. ${ }^{k} P<0.05$ vs Ang II+ZnPP IX. 
treated with Ang II (Figure 5B, 2C). Moreover, the ERK1/2 pathway inhibitor PD98059 significantly blocked Ang IIenhanced ERK1/2 phosphorylation and inhibited cell proliferation (Figure 4, 5C), confirming involvement of the ERK1/2 pathway in the HUVSMC growth response to Ang II.

\section{Discussion}

The excessive growth of VSMCs is a crucial event in the development of cardiovascular diseases such as atherosclerosis and hypertension. Among many factors implicated in the proliferation of VSMCs, Ang II appears to be one of the most impor$\tan t^{[21]}$. Therefore, the question of how to inhibit the proliferation of VSMCs exposed to chronic or long-term stimulation with Ang II has been the subject of therapeutic strategies for treatment of these diseases. Our study investigated the effect of neferine on the proliferation of VSMCs stimulated by Ang II. In the present study, we demonstrated that neferine has an antiproliferative effect on Ang II-induced VSMCs. The present data extend our knowledge of the action of neferine on vascular cells.

To explore the mechanism underlying the antiproliferative effect of neferine, we determined HO-1 expression in HUVSMCs. Data from previous studies suggested that induction of HO-1 could inhibit cell growth ${ }^{[12,22,23]}$. VSMCs from HO-1-deficient mice display enhanced DNA synthesis and cell growth ${ }^{[24]}$. Conversely, over-expression of HO-1 by either transfection of the HO-1 gene or exogenous administration of a certain HO-1 inducer reduces excessive VSMC proliferation $^{[25]}$. However, the effect of HO-1 on cell proliferation is highly variable and seems to be cell-type specific ${ }^{[8,26-28]}$, raising an important question of whether the antiproliferative effect of neferine on VSMC could be mediated via HO-1 expression. To test this, the effects on both HO-1 expression and growth inhibition of neferine were determined with the same concentration of neferine used in the present study. We found that the inhibitory effect of neferine on Ang II-stimulated HUVSMC proliferation was associated with a profound up-regulation of HO-1 protein expression in these cells. We also found that the inhibition of HUVSMC proliferation by neferine was partially, but not completely, reversed in the presence of ZnPP IX, an inhibitor of heme oxygenase activity. Likewise, the HO-1 inducer CoPP significantly prevented cell proliferation caused by Ang II, indicating a direct involvement of $\mathrm{HO}-1$ in the cell proliferation. Moreover, it is noteworthy that Ang II itself significantly reduced HO-1 expression; in contrast, neferine could significantly increase HO-1 expression in a concentrationdependent manner in HUVSMC. Thus, the above results demonstrated that the neferine-induced HO-1 expression might contribute, to a certain extent, to the antiproliferative effect of neferine on Ang II-stimulated HUVSMCs, which was in agreement with observations made in other cell lines ${ }^{[12,23]}$. Therefore, it is reasonable to speculate that HO-1 may be a potential target of the antiproliferative activity of neferine.

Ang II can stimulate VSMC proliferation as a mitogen ${ }^{[29]}$. Gradually, different signal transduction cascades have been implicated in Ang II-mediated VSMCs proliferation and migration, such as MAPK ${ }^{[30]}$ and PI3K/Akt ${ }^{[31,32]}$. Early in 2001, EI Mabrouk et al demonstrated that ERK1/2 phosphorylation is dose dependently increased by Ang II in VSMCs from spontaneously hypertensive (SHR) and Wistar-Kyoto (WKY) rats, and the ERK1/2 inhibitor PD-98059 can block Ang IIinduced VSMC growth ${ }^{[21]}$. In VSMCs, Ang II type I receptorinduced ERK1/2 activation occurs via $\mathrm{Ca}^{2+}$ and Src-dependent transactivation of the EGFR ${ }^{[33,34]}$. Ang II can activate ERK1/2 by the Ras/PKCzeta/MEK pathway in VSMC ${ }^{[35]}$. Therefore, the mechanism of ERK1/2 activation by Ang II potentially involves multiple signaling pathways. In this study, we investigated the relationship between Ang II-induced VSMC proliferation and ERK1/2 activation specifically. The results of our study showed that Ang II increased phosphorylation of ERK1/2 and stimulated cellular proliferation; in contrast, the ERK inhibitor PD98095 blocked Ang II-induced ERK1/2 phosphorylation and significantly inhibited Ang II-induced HUVSMC proliferation, indicating that ERK1/2 activation works in parallel with HUVSMC proliferation. These results are in line with EI Mabrouk et al and are also supported by another study in which VSMC proliferation induced by PDGF was inhibited by corynoxeine through the blocking of ERK1/2 phosphorylation ${ }^{[21,36]}$. Therefore, the present data confirm that activation of ERK1/2 plays an important part in HUVSMC proliferation induced by mitogens, such as Ang II.

Recently, Schwer et al observed that the curcumin-induced decrease in ERK1/2 phosphorylation was paralleled by HO-1 up-regulation and could be prevented by administration of the $\mathrm{HO}$ inhibitor $\mathrm{SnPP}^{[12]}$. In the present study, the results showed that neferine significantly reduced the Ang II-induced phosphorylation of ERK1/2 and cellular proliferation, and both effects could be attenuated in the presence of the HO inhibitor ZnPP IX. The ERK inhibitor PD98095 could also decrease Ang II-induced phosphorylation of ERK1/2 and inhibit cellular proliferation; more importantly, PD98095 prevented the increase in cell proliferation induced by ZnPP IX (Figure 4) and significantly reversed ZnPP IX-enhanced ERK $1 / 2$ phosphorylation (Figure 5C), thus confirming that the effect of HO-1 on HUVSMC proliferation involved the ERK1/2 pathway. Our findings are similar to that of Schwer et al ${ }^{[12]}$, suggesting that HO-1 up-regulation suppresses the ERK1/2 signaling pathway. Based on the above data, our study reveals that neferine may act by at least partially up-regulating HO-1, leading to the down-regulation of ERK1/2 phosphorylation to inhibit Ang II-induced HUVSMC proliferation.

It has been reported that the up-regulation of the cyclindependent kinase inhibitor $\mathrm{p} 21^{\mathrm{WAF} 1 / \mathrm{CIP1}}$ is involved in the antiproliferative effect of $\mathrm{HO}-1^{[25,37]}$. $\mathrm{CO}$ is one of the products of heme degradation. The antiproliferative effect of YS 49 on VSMC is reversed by pretreatment with hemoglobin, a $\mathrm{CO}$ scavenger ${ }^{[38]}$. Cdk-2 is a key regulator of both $\mathrm{G}_{1}$ and $\mathrm{S}$ phase cell progression. Duckers et al have demonstrated that $\mathrm{CO}$ induces the expression of the cdk-2 inhibitor $\mathrm{p} 21^{\mathrm{WAF} 1 / \mathrm{CIP} 1}$, suggesting that $\mathrm{CO}$ may block cdk-2 activity via multiple mechanisms to inhibit VSMC proliferation ${ }^{[8]}$. In addition, Ang II induces ROS production through the ERK1/2 pathway 
in VSMCs ${ }^{[39]}$. Oxidants, including ROS, are signals involved in the regulation of cell proliferation ${ }^{[40]}$. Results from Taille et al emphasize the concept that the antioxidant properties of the HO-bilirubin pathway are related not only to its ROS scavenging properties but also to the modulation of ROS production $^{[27]}$. According to the above studies, although we have shown that HO-1 up-regulation by neferine participated in the inhibition of HUVSMC proliferation via suppression of ERK1/2 activation, we did not exclude the possibility that neferine may also induce the expression of other antiproliferative enzymes or that the effect of neferine may be achieved through the tightly regulated process of the multiple pathways that may be activated or inactivated by neferine. For example, HO- 1 can act both as a sensor to and as a target of redox-based mechanisms involving nitric oxide (NO) in rat $\mathrm{H} 9 \mathrm{c} 2$ cells ${ }^{[41]}$. NO plays a significant role in transmembrane signaling in the ischemic myocardium; it activates $\mathrm{HO}$, which further stimulates the production of cGMP, presumably by $\mathrm{CO}$ signaling. Thus, NO not only potentiates cGMP-mediated intracellular signaling but also functions as a retrograde messenger for $\mathrm{CO}$ signaling in the heart ${ }^{[42]}$. In fact, we also found in this study that Ang II caused a significant decrease in the production of NO in HUVSMC; neferine had no significant effect on NO production in cells in the absence of Ang II, whereas neferine exhibited a significant up-regulating effect on the production of NO in cells in the presence of Ang II (data not shown). Ang II activates membrane NAD(P)H oxidases in VSMCs to produce ROS, which are involved in the pleiotropic effects of Ang $\mathrm{II}^{[2]}$. One of the most well established consequences of superoxide generated by Ang II is the inactivation of NO in $\mathrm{VSMCs}^{[43,44]}$. Thus, it remains uncertain whether the antiproliferative action of neferine could also be mediated by increasing NO production or reducing ROS production.

In summary, we demonstrated that neferine is capable of inhibiting Ang II-induced HUVSMC proliferation. Therefore, neferine might be a promising pharmacological agent for the prevention and treatment of vascular obstructive diseases associated with excessive VSMC proliferation, such as hypertension and atherosclerosis.

\section{Acknowledgements}

This study was supported by the Administration of Traditional Chinese Medicine of Zhejiang Province (№ 2007CA112).

\section{Author contribution}

Peng CHEN designed the research; Xiao-chun LI and Guo-xin TONG performed the research; Yu ZHANG, Shan-xin LIU, Qihui JIN, and Huai-hong CHEN analyzed the data; Xiao-chun LI, and Peng CHEN wrote the paper.

\section{Abbreviations}

MTT, 3-(4,5-dimethylthiazol-2-yl)-2,5-diphenyltetrazolium bromide; CoPP, cobalt-protoporphyrin; ERK, extracellular signalregulated kinase; HO, heme oxygenase; PD98059, 2'-amino-3'methoxyflavone; MAPK, mitogen-activated protein kinase; MEK, MAP kinase; ZnPP, zinc protoporphyrin; GAPDH, glyceraldehyde 3-phosphate dehydrogenase; Ang, angiotensin; HUVSMC, human umbilical vascular smooth muscle cell; DMEM, Dulbecco's modified Eagle's medium; ROS, reactive oxygen species; Nef, neferine; JNK, Jun N-terminal kinase; SnPP, tin protoporphyrin; EGFR, epidermal growth factor receptor; PDGF, platelet-derived growth factor; cdk-2, cyclin-dependent kinase-2.

\section{References}

1 Touyz RM, Schiffrin EL. Signal transduction mechanisms mediating the physiological and pathophysiological actions of angiotensin II in vascular smooth muscle cells. Pharmacol Rev 2000; 52: 639-72.

2 Mehta PK, Griendling KK. Angiotensin II cell signaling: physiological and pathological effects in the cardiovascular system. Am J Physiol Cell Physiol 2007; 292: C82-97.

3 Yin G, Yan C, Berk BC. Angiotensin II signaling pathways mediated by tyrosine kinases. Int J Biochem Cell Biol 2003; 35: 780-3.

4 Suzuki H, Motley ED, Frank GD, Utsunomiya H, Eguchi S. Recent progress in signal transduction research of the angiotensin II type1 receptor: protein kinases, vascular dysfunction and structural requirement. Curr Med Chem Cardiovasc Hematol Agents 2005; 3 : 305-22.

5 Graf K, Xi XP, Yang D, Fleck E, Hsueh WA, Law RE. Mitogen-activated protein kinase activation is involved in platelet-derived growth factordirected migration by vascular smooth muscle cells. Hypertension 1997; 29: 334-9.

6 Wu L, Wang R. Carbon monoxide: endogenous production, physiological functions, and pharmacological applications. Pharmacol Rev 2005; 57: 585-630.

7 Keyse SM, Tyrrell RM. Heme oxygenase is the major 32-kDa stress protein induced in human skin fibroblasts by UVA radiation, hydrogen peroxide, and sodium arsenite. Proc Natl Acad Sci USA 1989; 86: 99-103.

8 Duckers HJ, Boehm M, True AL, Yet SF, San H, Park JL, et al. Heme oxygenase-1 protects against vascular constriction and proliferation. Nat Med 2001; 7: 693-8.

9 Deng YM, Wu BJ, Witting PK, Stocker R. Probucol protects against smooth muscle cell proliferation by upregulating heme oxygenase-1. Circulation 2004; 110: 1855-60.

10 Lee TS, Chang CC, Zhu Y, Shyy JY. Simvastatin induces heme oxygenase-1: a novel mechanism of vessel protection. Circulation 2004; 110: 1296-302.

11 Chang T, Wu L, Wang R. Inhibition of vascular smooth muscle cell proliferation by chronic hemin treatment. Am J Physiol Heart Circ Physiol 2008; 295: H999-H1007.

12 Schwer $\mathrm{Cl}$, Guerrero AM, Humar M, Roesslein M, Goebel U, Stoll P, et al. Heme oxygenase-1 inhibits the proliferation of pancreatic stellate cells by repression of the extracellular signal-regulated kinase $1 / 2$ pathway. J Pharmacol Exp Ther 2008; 327: 863-71.

13 Cao JG, Tang XQ, Shi SH. Multidrug resistance reversal in human gastric carcinoma cells by neferine. World J Gastroenterol 2004; 10 : 3062-4.

14 Chen J, Qi J, Chen F, Liu JH, Wang T, Yang J, et al. Relaxation mechanisms of neferine on the rabbit corpus cavernosum tissue in vitro. Asian J Androl 2007; 9: 795-800.

15 Feng $\mathrm{Y}$, Wu J, Cong R, Wang C, Zong Y, Feng Z. The effect of neferine on foam cell formation by anti-low density lipoprotein oxidation. J Tongii Med Univ 1998; 18: 134-6.

$16 \mathrm{Yu} \mathrm{J}$, Hu WS. Effects of neferine on platelet aggregation in rabbits. Yao Xue Xue Bao 1997; 32: 1-4.

17 Qian JQ. Cardiovascular pharmacological effects of bisbenzyliso- 
quinoline alkaloid derivatives. Acta Pharmacol Sin 2002; 23: 1086 92.

18 Burnette WN. "Western blotting": electrophoretic transfer of proteins from sodium dodecyl sulfate - polyacrylamide gels to unmodified nitrocellulose and radiographic detection with antibody and radioiodinated protein A. Anal Biochem 1981; 112: 195-203.

19 van de Loosdrecht AA, Beelen RH, Ossenkoppele GJ, Broekhoven MG, Langenhuijsen MM. A tetrazolium-based colorimetric MTT assay to quantitate human monocyte mediated cytotoxicity against leukemic cells from cell lines and patients with acute myeloid leukemia. J Immunol Methods 1994; 174: 311-20.

20 Pozarowski P, Darzynkiewicz Z. Analysis of cell cycle by flow cytometry. Methods Mol Biol 2004; 281: 301-11.

21 El Mabrouk M, Touyz RM, Schiffrin EL. Differential ANG Il-induced growth activation pathways in mesenteric artery smooth muscle cells from SHR. Am J Physiol Heart Circ Physiol 2001; 281: H30-9.

22 Pae HO, Jeong GS, Jeong SO, Kim HS, Kim SA, Kim YC, et al. Roles of heme oxygenase-1 in curcumin-induced growth inhibition in rat smooth muscle cells. Exp Mol Med 2007; 39: 267-77.

23 Woo SW, Lee SH, Ko G, Kim YC, Sohn DH. Isoliquiritigenin inhibits cell proliferation by a heme oxygenase-dependent pathway in rat hepatic stellate cells. Planta Med 2008; 74: 834-9.

24 Yet SF, Layne MD, Liu X, Chen YH, Ith B, Sibinga NE, et al. Absence of heme oxygenase-1 exacerbates atherosclerotic lesion formation and vascular remodeling. Faseb J 2003; 17: 1759-61.

25 Juan SH, Lee TS, Tseng KW, Liou JY, Shyue SK, Wu KK, et al. Adenovirus-mediated heme oxygenase- 1 gene transfer inhibits the development of atherosclerosis in apolipoprotein E-deficient mice. Circulation 2001; 104: 1519-25.

26 Marinissen MJ, Tanos T, Bolos M, de Sagarra MR, Coso OA, Cuadrado A. Inhibition of heme oxygenase-1 interferes with the transforming activity of the Kaposi sarcoma herpesvirus-encoded $G$ protein-coupled receptor. J Biol Chem 2006; 281: 11332-46.

27 Taille C, Almolki A, Benhamed M, Zedda C, Megret J, Berger P, et al. Heme oxygenase inhibits human airway smooth muscle proliferation via a bilirubin-dependent modulation of ERK1/2 phosphorylation. J Biol Chem 2003; 278: 27160-8.

28 Li Volti G, Wang J, Traganos F, Kappas A, Abraham NG. Differential effect of heme oxygenase- 1 in endothelial and smooth muscle cell cycle progression. Biochem Biophys Res Commun 2002; 296: 1077 82.

29 Weber H, Taylor DS, Molloy CJ. Angiotensin II induces delayed mitogenesis and cellular proliferation in rat aortic smooth muscle cells. Correlation with the expression of specific endogenous growth factors and reversal by suramin. J Clin Invest 1994; 93: 788-98.

30 Seshiah PN, Weber DS, Rocic P, Valppu L, Taniyama Y, Griendling KK. Angiotensin II stimulation of $\mathrm{NAD}(\mathrm{P}) \mathrm{H}$ oxidase activity: upstream mediators. Circ Res 2002; 91: 406-13.

31 Saward L, Zahradka P. Angiotensin II activates phosphatidylinositol 3-kinase in vascular smooth muscle cells. Circ Res 1997; 81: 249-
57.

32 Chiou WF, Chen CC, Wei BL. 3,4-Di-O-caffeoylquinic acid inhibits angiotensin-ii-induced vascular smooth muscle cell proliferation and migration by downregulating the jnk and pi3k/akt signaling pathways. Evid Based Complement Alternat Med 2009. doi: 10.1093/ecam/ nep140.

33 Bokemeyer D, Schmitz U, Kramer HJ. Angiotensin II-induced growth of vascular smooth muscle cells requires an Src-dependent activation of the epidermal growth factor receptor. Kidney Int 2000; 58: 549-58.

34 Eguchi S, Numaguchi K, Iwasaki H, Matsumoto T, Yamakawa T, Utsunomiya $\mathrm{H}$, et al. Calcium-dependent epidermal growth factor receptor transactivation mediates the angiotensin II-induced mitogenactivated protein kinase activation in vascular smooth muscle cells. J Biol Chem 1998; 273: 8890-6.

35 Zhao Y, Liu J, Li L, Liu L, Wu L. Role of Ras/PKCzeta/MEK/ERK1/2 signaling pathway in angiotensin II-induced vascular smooth muscle cell proliferation. Regul Pept 2005; 128: 43-50.

$36 \mathrm{Kim} \mathrm{TJ}$, Lee JH, Lee JJ, Yu JY, Hwang BY, Ye SK, et al. Corynoxeine isolated from the hook of Uncaria rhynchophylla inhibits rat aortic vascular smooth muscle cell proliferation through the blocking of extracellular signal regulated kinase $1 / 2$ phosphorylation. Biol Pharm Bull 2008; 31: 2073-8.

37 Notoya M, Nishimura H, Woo JT, Nagai K, Ishihara Y, Hagiwara H. Curcumin inhibits the proliferation and mineralization of cultured osteoblasts. Eur J Pharmacol 2006; 534: 55-62.

38 Sun JJ, Kim HJ, Seo HG, Lee JH, Yun-Choi HS, Chang KC. YS 49, 1-(alpha-naphtylmethyl)-6,7-dihydroxy-1,2,3,4-tetrahydroisoquinoline, regulates angiotensin II-stimulated ROS production, JNK phosphorylation and vascular smooth muscle cell proliferation via the induction of heme oxygenase-1. Life Sci 2008; 82: 600-7.

39 Kim JE, Kang YJ, Lee KY, Choi HC. Isoproterenol inhibits angiotensin II-stimulated proliferation and reactive oxygen species production in vascular smooth muscle cells through heme oxygenase-1. Biol Pharm Bull 2009; 32: 1047-52.

40 Wedgwood S, Black SM. Role of reactive oxygen species in vascular remodeling associated with pulmonary hypertension. Antioxid Redox Signal 2003; 5: 759-69.

41 Naughton P, Foresti R, Bains SK, Hoque M, Green CJ, Motterlini R. Induction of heme oxygenase 1 by nitrosative stress. A role for nitroxyl anion. J Biol Chem 2002; 277: 40666-74.

42 Maulik N, Engelman DT, Watanabe M, Engelman RM, Das DK. Nitric oxide - a retrograde messenger for carbon monoxide signaling in ischemic heart. Mol Cell Biochem 1996; 157: 75-86.

43 Gryglewski RJ, Palmer RM, Moncada S. Superoxide anion is involved in the breakdown of endothelium-derived vascular relaxing factor. Nature 1986; 320: 454-6.

44 Rubanyi GM, Vanhoutte PM. Superoxide anions and hyperoxia inactivate endothelium-derived relaxing factor. Am J Physiol 1986; 250: H822-7. 